\title{
Thermal conductivity of graphene mediated by strain and size
}

\author{
Youdi Kuang a,b, c ${ }^{\text {, Lucas Lindsay }}{ }^{\mathrm{d}}$, Sanqiang Shi ${ }^{\mathrm{c}, \mathrm{e}}{ }^{*}$, Xinjiang Wang ${ }^{\mathrm{a}}$, and Baoling \\ Huang
}

${ }^{a}$ College of Science and Engineering, Jinan University, Guanzhou, China

${ }^{\mathrm{b}}$ Department of Mechanical and Aerospace Engineering, The Hong Kong University of Science and Technology, Clear Water Bay, Kowloon, Hong Kong

${ }^{\mathrm{c}}$ Department of Mechanical Engineering, the Hong Kong Polytechnic University, Hung Hom, Kowloon, Hong Kong

${ }^{\mathrm{d}}$ Materials Science and Technology Division, Oak Ridge National Laboratory, Oak Ridge, Tennessee 37831, USA.

${ }^{\mathrm{e}}$ Hong Kong Polytechnic University Shenzhen Research Institute, Shenzhen, China

\section{Abstract}

Based on first-principles calculations and full iterative solution of the linearized Boltzmann-Peierls transport equation for phonons, we systematically investigate effects of strain, size and temperature on the thermal conductivity $k$ of suspended graphene. The calculated size-dependent and temperature-dependent $k$ for finite samples agree well with experimental data. The results show that, contrast to the convergent room-temperature $k=5450 \mathrm{~W} / \mathrm{m}-\mathrm{K}$ of unstrained graphene at a sample size $\sim 8 \mathrm{~cm}, k$ of strained graphene diverges with increasing the sample size even at high temperature. Out-of-plane acoustic phonons are responsible for the significant size effect in unstrained and strained graphene due to their ultralong mean free path

\footnotetext{
* Correspondence should be addressed to:

Dr. Sanqiang Shi, Tel: 852-27667821, E-mail: san.qiang.shi@ polyu.edu.hk or

Dr. Baoling Huang, Tel: 852-23587181, E-mail: mebhuang@ ust.hk.
} 
and acoustic phonons with wavelength smaller than $10 \mathrm{~nm}$ contribute $80 \%$ to the intrinsic room temperature $k$ of unstrained graphene. Tensile strain hardens the flexural modes and increases their lifetimes, causing interesting dependence of $k$ on sample size and strain due to the competition between boundary scattering and intrinsic phonon-phonon scattering. $k$ of graphene can be tuned within a large range by strain for the size larger than $500 \mu \mathrm{m}$. These findings shed light on the nature of thermal transport in two-dimensional materials and may guide predicting and engineering $k$ of graphene by varying strain and size.

Keywords: phonon thermal transport, graphene, first principles, strain and size effects

\section{Introduction}

Graphene, a two-dimensional (2D) sheet of carbon atoms, has attracted great interest in recent years due to its extraordinary mechanical, chemical, electronic and thermal properties. The experimentally reported ultrahigh thermal conductivity $k$ (up to $5300 \mathrm{~W} / \mathrm{m}-\mathrm{K}$ ) [1] of graphene renders it the most thermally conductive material, and of promise for thermal management applications [2-4]. Moreover, graphene provides a benchmark model for the study of thermal transport in 2D materials. Therefore, intensive efforts have been committed to understand the underlying thermal transport physics in graphene experimentally [1, 5-16] and theoretically [2, 17-30].

Previous experimental studies $[1,5,6,10]$ using an opto-thermal Raman technique have reported $k$ values of suspended graphene that scatter significantly, 
ranging from 600 [10] to 5300 [31] W/m-K even with temperature effects accounted for. Using direct thermal-bridge measurements, $\mathrm{Xu}$ et al. [7] recently observed significant size effects on $k$ of graphene and reported that $k$ diverges logarithmically with sample length; in contrast, previous Raman experiments did not discern a sizedependence of $k[1,6]$. Pettes et al. [11] found that $k$ of graphene may be significantly influenced by the residual polymeric layer produced during the transfer process of graphene. It can be seen from previous experiments that measured $k$ depends strongly on various extrinsic factors including sample size, process conditions, sample quality, measurement method and substrate coupling [12, 13], all giving large scatter in experimental $k$ data.

On the theoretical side, fundamental problems concerning the details of thermal transport in graphene have been subjects of debate [17-30], including the convergence behavior of $k$ with system size, the extent of the diffusive and ballistic transport regimes, the role of flexural acoustic (ZA) phonons for thermal transport and strain effects on the convergence of $k$. It is generally believed that acoustic phonons [31] dominate the thermal transport in graphene. Based on this, 2D models give a logarithmic divergence with system size [27] but neglect the contributions from ZA phonons due to their low group velocities near the center of first Brillouin zone (FBZ) and their large Grüneisen parameters [32]. However, molecular dynamics (MD) simulations $[17,18,26,29,30]$ with large system sizes have reported that roomtemperature $k$ of graphene converges, though at values much lower than experimental results on finite-size systems. 
First-principles lattice dynamics calculations $[22,24,28]$ of $k$ of graphene within three-phonon scattering framework have also been conducted. Using the single-mode relaxation time approximation (SMRTA), Bonini et al. [22] showed that $k$ of infinite graphene diverges under infinitesimal isotropic tensile strains, while $k$ converges to $\sim 550 \mathrm{~W} / \mathrm{m}-\mathrm{K}$ for infinite unstrained graphene at room temperature. The results are partly different with aforementioned MD predictions [18] that $k$ of infinite graphene diverges only under large tensile strain (> 0.02). We note that the SMRTA incorrectly treats the momentum-conserving Normal $(\mathrm{N})$ processes as independent resistive processes on the same footing as Umklapp (U) processes [33], and it cannot be used to appropriately present the phonon thermal transport in graphene, as justified by Lindsay et al. [23] and Fugallo et al. [28]. Based on a full iterative solution of the linearized Boltzmann-Peierls Equation (BPE), Lindsay et al. [23] found that ZA phonons give the dominant contributions to $k$ in finite graphene up to $50 \mu \mathrm{m}$ with strong dependence of $k$ on boundary scattering; Fugallo et al. [28] found that room temperature $k$ of unstrained graphene with a size of $1000 \mu \mathrm{m}$ is up to $4300 \mathrm{~W} / \mathrm{m}-\mathrm{K}$, close to the reported highest experimental value [31]. Although the previous works provided excellent insights into understanding thermal transport of graphene, many conclusions apply to finite graphene because of the compulsively adopted boundary constraint. It can be seen clearly from the literature that the following fundamental problems still remain unclear: 1) $k$ convergence in strained graphene by full solutions of BPE; 2) the role of ZA modes in $k$ of strained graphene at different temperatures; 3 ) the scattering behavior of low-frequency phonons at the long-wavelength limit; 4) the interplay of strain and size effects. 
In this work, we intend to elucidate these problems using a rigorous first principles BPE for phonon transport approach with and without applying boundary constraint. Full iterative solutions of the linearized BPE from reciprocal-space calculations and further mathematical analysis show that with increasing system size $k$ converges for unstrained graphene and diverges for strained graphene. Mode contribution analysis shows that ZA phonons are the major heat carriers and control the convergence behaviors in both unstrained and strained graphene up to $3000 \mathrm{~K}$. Further, the long mean free paths of ZA phonons make finite size effects on $k$ persistent up to $\sim 8 \mathrm{~cm}$ for unstrained graphene. The joint effect of strain and size on $k$ of finite graphene is also clarified, which makes it promising to tune $k$ of graphene in a large range when the sample size is larger than $500 \mu \mathrm{m}$. Considering the large graphene fracture strain $(\sim 0.25)$ and recently successfully fabrication [34] of single crystal graphene sample with the size of a few centimeters, mediating $k$ of graphene by tensile strain and size is of practical significance.

\section{Computational methodologies}

A microscopic description of the lattice thermal conductivity $k$ can be derived from BPE for phonons $[21,22,24,35]$ within the three-phonon scattering framework. Considering the isotropic thermal conductivity of infinite graphene, along an in-plane crystallographic direction $\alpha$, the intrinsic $k$ is calculated by the following equation [23, 35]:

$$
k=k^{\alpha \alpha}=\sum_{\lambda} k_{\lambda}=\frac{1}{k_{\mathrm{B}} T^{2} V N_{1}^{2}} \sum_{\lambda} f_{\lambda}\left(f_{\lambda}+1\right)\left(\mathrm{h} \omega_{\lambda}\right)^{2} v_{\lambda}^{\alpha} v_{\lambda}^{\alpha} \tau_{\lambda},
$$


where $\lambda$ represents a phonon mode with wavevector $\mathbf{q}$ and branch index $j . k_{\lambda}$ is the contribution of mode $\lambda$ to $k . \omega_{\lambda}, v_{\lambda}^{\alpha}$, and $\tau_{\lambda}$ are the angular frequency, group velocity and phonon lifetime, respectively. $k_{\mathrm{B}}, \hbar, f_{\lambda}$ are the Boltzmann constant, the reduced Plank constant and the Bose-Einstein distribution of phonons at temperature $T$, respectively. $V$ is the volume of the graphene unit cell with a thickness of $0.335 \mathrm{~nm}$ [23]. This work combines an iteratively self-consistent solution to the linearized BPE with harmonic and anharmonic interatomic force constants (IFCs) from Density Functional Perturbation Theory (DFPT) and Density Functional Theory (DFT) calculations [23, 24], respectively, using the QUANTUM ESPRESSO package [36] within the local density approximation (LDA) and using a norm-conserving pseudopotential to represent the core carbon electrons. Before calculating IFCs, the 2atom unit cell of unstrained graphene is optimized fully along three axial directions to minimize the internal stress. The convergence precisions for energy and atomic force are taken as $1 \times 10^{-10} \mathrm{eV}$ and $1 \times 10^{-7} \mathrm{eV} / \AA$, respectively, to ensure the absolute value of in-plane stress is less than $0.01 \mathrm{kbar}$. To determine the harmonic IFCs, DFPT calculations are employed with a $13 \times 13 k$-point mesh and 120 Ryd plane-wave cutoff for the 2-atom unit cell. To determine the interatomic forces and resulting anharmonic IFCs, DFT calculations with $\Gamma$-point sampling in slightly perturbed $162-$ atom supercells with a 100 Ryd plane-wave cutoff are used. For harmonic IFCs of unstrained graphene, the translational and rotational invariance conditions are enforced using the acoustic sum rules proposed in the work by Bonini et al.[22]. Only translational invariance conditions are applied to harmonic IFCs of strained graphene. 
For anharmonic IFCs, translational invariance is enforced via a $\chi^{2}$ fitting procedure described in Ref. [37]. We note that the accuracy of these anharmonic IFCs was tested and discussed in Ref. [37]. Further technical details for the calculations of dispersion relations and three-phonon scattering rates can be found elsewhere [23, 35]. In order to judge the convergence of intrinsic $k$ at large grid density $\left(N_{1}>200\right)$, a modified ShengBTE code [35] is used to calculate $k$ iteratively based on these IFCs. Comparison of the calculated dispersion of graphene with experimental data [38, 39] gives excellent agreements. The full 2D FBZ (Figure 1a) is discretized into a $\Gamma$ centered regular $N_{1} \times N_{1}$ grid with $N_{1}$ up to 501 considered in this study.

\section{Results and Discussion}

\subsection{Intrinsic thermal conductivities of unstrained and strained graphene}

Figure 1a shows the calculated room-temperature $k$ of unstrained graphene (isotropic tensile strain $\varepsilon=0)$ with respect to the $q$-point sampling density. Here, $\varepsilon=(a$ $\left.a_{0}\right) / a_{0}$ where $a_{0}=2.44 \AA$ is the calculated equilibrium lattice constant and $a$ is the lattice constant for a given tensile strain. For each $N_{1}$, an iteration precision of $1 \times 10^{-5}$ (difference of $k$ values for successive iterative steps) is taken to ensure full selfconsistent convergence of $k$. Interestingly, $k$ decreases with increasing number of modes and a grid-converged $k=5450 \mathrm{~W} / \mathrm{m}-\mathrm{K}$ is achieved for $N_{1} \geq 301$. This $k$ value from the iterative approach is several-fold higher than those from the SMRTA [22, 24], confirming previous findings [21, 23] that both $\mathrm{N}$ - and U-processes and their relationship influencing the nonequilibrium populations of phonon modes are 

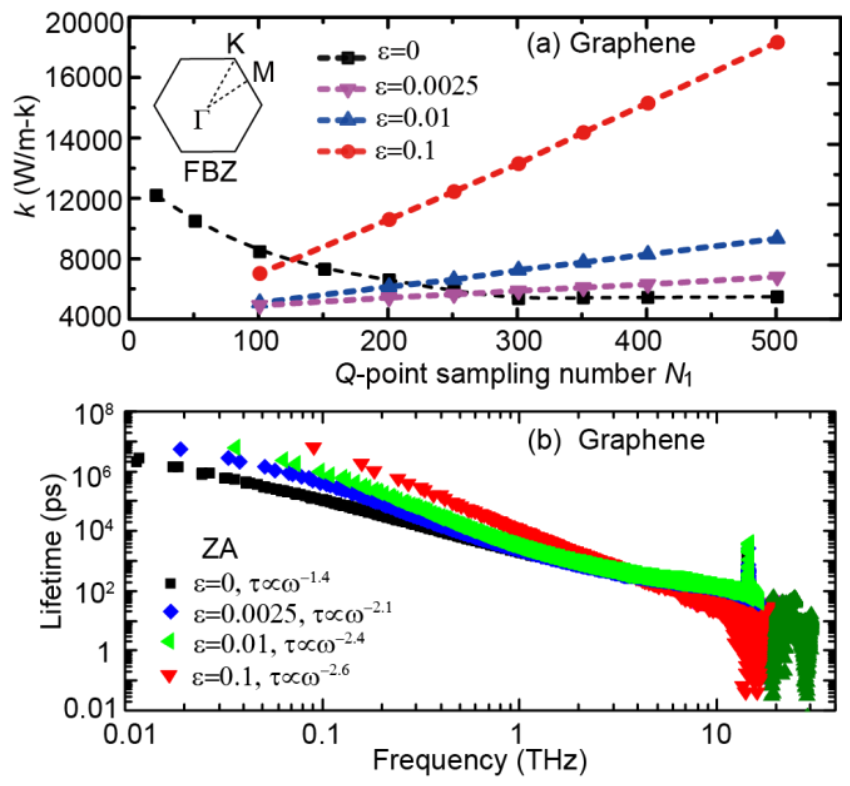

Fig. 1. (a) Convergence of $k$ of graphene with $q$-point sampling density for different isotropic tensile strains. (b) Lifetimes of ZA phonons in graphene under different strains.

important for determining $k$ of graphene. The convergence of $k$ in our calculations for infinite unstrained graphene also justifies that intrinsic three-phonon scatterings can confine $k$, i.e., higher-order inter-phonon scatterings are not required for convergence of $k$ as was previously suggested for $k$ of unstrained single-walled carbon nanotubes $[40,41]$. Under different strains $\varepsilon=0.0025,0.01$ and $0.1, k$ increases nearly linearly with increasing $N_{1}$ and at a fixed $N_{1}$ a larger $\varepsilon$ gives a higher $k$, indicating nonnegligible contributions from longer-wavelength phonon modes and the divergence of $k$ with increasing system size under strain. Unlike the work of Ref. [22], anharmonic IFCs are calculated here for each strain value considered. With increasing strain the magnitudes of the anharmonic IFCs tend to decrease. Our calculations show that neglecting strain effects on these anharmonic IFCs does not change the convergence 
behavior qualitatively but causes significant underestimation of $k$ especially for large strain. For example, a 31-fold underestimation in $k$ at $\varepsilon=0.1$ for $N_{1}=301$ is observed when using unstrained anharmonic IFCs. The mixed IFC tests justify that the linearization of ZA dispersion plays a key role for the $k$ divergence in strained graphene. Unless specified otherwise, all the results shown below are for $N_{1}=301$ for unstrained and strained graphene.

Figure $1 \mathrm{~b}$ shows the calculated transport lifetimes $\tau_{\lambda}$ of ZA acoustic phonons for strained and unstrained infinite graphene at room temperature. Here we only consider ZA phonons since they dominate thermal transport in graphene. For unstrained graphene, $\tau_{\lambda} \propto \omega_{\lambda}^{-1.4}$ is found for low-frequency ZA phonons. In strained graphene, long wavelength ZA phonon lifetimes are more strongly dependent on frequency, having $\tau_{\lambda} \propto \omega_{\lambda}^{-2.1}, \omega_{\lambda}^{-2.4}$ and $\omega_{\lambda}^{-2.6}$ for $\varepsilon=0.0025,0.01$ and 0.1 , respectively. Two factors may contribute to the lifetime enhancement at a given low frequency by tensile strains: one is that increasing strain decreases the magnitude of the anharmonic IFCs [23] and consequently reduces the scattering matrix elements; the other is zone center dispersion linearization of ZA modes [22] reduces ZA phonon density and consequently gives rise to less scatterings of ZA phonons [18]. The reduction of ZA phonon density is a result of linearization. The strain dependent phonon dispersion relations are shown in Fig. 2. In an early work by Bonini et al. [22], the authors found quadratic ZA dispersion after enforcing translational and rotational invariance conditions on harmonic IFCs of unstrained graphene from DFPT calculations, and found that ZA dispersion is linearized after applying tensile strains. More recently, 
Carrete et al.[43] proposed the 'by construction' approach to build the physical IFCs and also suggested the quadratic ZA dispersion for unstrained graphene. The abrupt ZA dispersion transition from purely quadratic to linearized at the long-wavelength limit has been shown via the elastic theory of a thin plate [42]. The linearization degree is more significant with increasing strain level as shown in the inset.

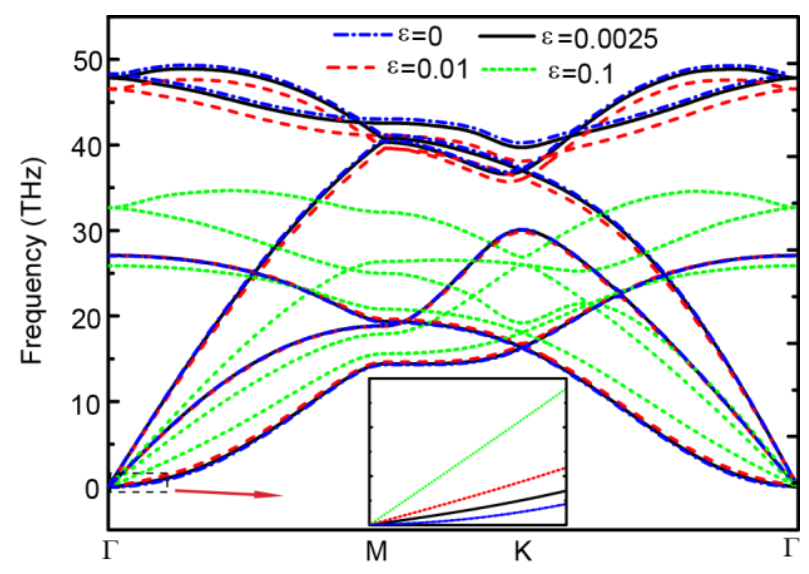

Fig.2. The calculated phonon dispersion relations of graphene under different tensile strains. The inset shows the ZA dispersion linearization at low frequencies.

These lifetime data provide a better understanding of the results shown in Figure 1a. The nonzero minimum $|q|$ corresponding to $N_{1}$ is $|q|=\frac{\gamma}{N_{1}}$ where $\gamma$ is a constant. Then, at small wavevector the phonon frequency and velocity corresponding to $|q|$ in the ZA branch [22] are given by $\omega_{\lambda}^{2}=C|q|^{2}+D|q|^{4}=\frac{C \gamma^{2}}{N_{1}{ }^{2}}+\frac{D \gamma^{4}}{N_{1}{ }^{4}}$ and $v_{\lambda}^{\alpha}=\frac{d \omega_{\lambda}}{d q}$. The lifetimes follow a power law behavior $\tau_{\lambda}=A \omega_{\lambda}^{-\beta}$. Here $A, C, D$ and $\beta$ are straindependent parameters and $C=0$ for zero strain. For unstrained and strained graphene 
as $N_{1} \rightarrow \infty, \omega_{\lambda} \rightarrow 0$ and $f_{\lambda} \rightarrow \frac{k_{\mathrm{B}} T}{\hbar \omega_{\lambda}}$. Therefore, for ZA mode $\lambda$ in unstrained graphene $(\beta=1.4)$, we have $\lim _{\substack{\omega_{\lambda} \rightarrow 0 \\ N_{1} \rightarrow \infty}} k_{\lambda} \propto \lim _{N_{1} \rightarrow \infty} \frac{1}{N_{1}^{4-2 \beta}}=0$ based on Eq.(1), implying ZA phonons in the long-wavelength limit contribute little to $k$ and $k$ will converge. For strained graphene $(\beta>2)$, we have $\lim _{\substack{\omega_{\lambda} \rightarrow 0 \\ N_{1} \rightarrow \infty}} k_{\lambda} \propto \lim _{N_{1} \rightarrow \infty} N_{1}^{\beta-2} \rightarrow \infty$, indicating $k$ will diverge. The fitted $\beta$ parameters in our calculations support the corresponding conclusions regarding the convergence of unstrained and strained graphene. Further, our calculations with larger $N_{1}\left(N_{1}=401,501\right)$ gives slightly larger $\beta$ values at low frequencies than that corresponding to $N_{1}=301$ for graphene with strain $\varepsilon=0.01$. The reason is as follows: larger $N_{1}$ involves lower frequencies for which the dispersion relations have stronger linearization than those of high-frequency phonons. Therefore, the corresponding $\beta$ value based on these lower frequencies is larger, and still gives divergent behavior. Compared with the results shown in Fig.1 from discrete numerical solutions of the BPE, the present analytical expressions based on the $\beta$ behavior more clearly demonstrate the strain dependent $k$ convergence. When $k$ is divergent, $k$ will keep increasing when more longer-wavelength modes are considered in BPE without boundary constraint. Therefore, a series of calculations with different large $q$-point grid densities are required to sample long-wavelength ZA modes at low frequencies to judge the $k$ divergence or convergence behavior.

\section{2 . Comparisons with previous experiments and phase space approach}


We also compare the calculated $k$ of unstrained graphene with measured data for graphene of varying sample size, temperature and isotope abundance. Here we discuss the implementation of extrinsic scattering mechanisms in the calculation of $k$ for better comparison with measured $k$ values. We define $k$ as a scalar value, ignoring anisotropy from finite system size due to the relatively large experimental samples that we are comparing with [23]. The lifetime $\tau_{\lambda}^{f}$ of a phonon in a finite sample may be calculated using the Matthiessen rule [23], expressed here as $\frac{1}{\tau_{\lambda}^{f}}=\frac{1}{\tau_{\lambda}}+\frac{1}{\tau_{\lambda}^{i s o}}+\frac{1}{\tau_{\lambda}^{b}}+\frac{1}{\tau_{\lambda}^{w}} \cdot \frac{1}{\tau_{\lambda}}$ is the intrinsic phonon-phonon scattering rate; $\frac{1}{\tau_{\lambda}^{i s o}}$ represents the scattering rate from naturally occurring isotopes $\left(1.1 \% \mathrm{C}^{13}\right)$ in graphene and is obtained from perturbation theory for a random isotope distribution [35]; $\frac{1}{\tau_{\lambda}^{b}}$ represents the scatting rate by contact boundaries and is expressed empirically as $\frac{1}{\tau_{\lambda}^{b}}=\frac{2\left|v_{\lambda}^{x}\right|}{L}[40]$ and $\frac{1}{\tau_{\lambda}^{b}}=\frac{\left|v_{\lambda}^{x}\right|}{D}[23,28]$ respectively for a rectangle sample and a circular sample. This is consistent with experiments using rectangular samples in the thermal-bridge measurements of $k$ [7] and circular samples in the Raman measurements of $k$ [6]. The direction of the temperature gradient, $x$, is assumed to be along the sample length, $L$, or diameter, $D . \frac{1}{\tau_{\lambda}^{w}}$ represents the scatting rate due to finite sample width $W$ of the rectangular samples and is expressed as [44, 45] $\frac{1}{\tau_{\lambda}^{w}}=\frac{2\left|v_{\lambda}^{y}\right|}{W}$, where $v_{\lambda}^{y}$ is the group velocity along the width direction and perpendicular to the transport direction. As shown in Figure 3a, the calculated room 

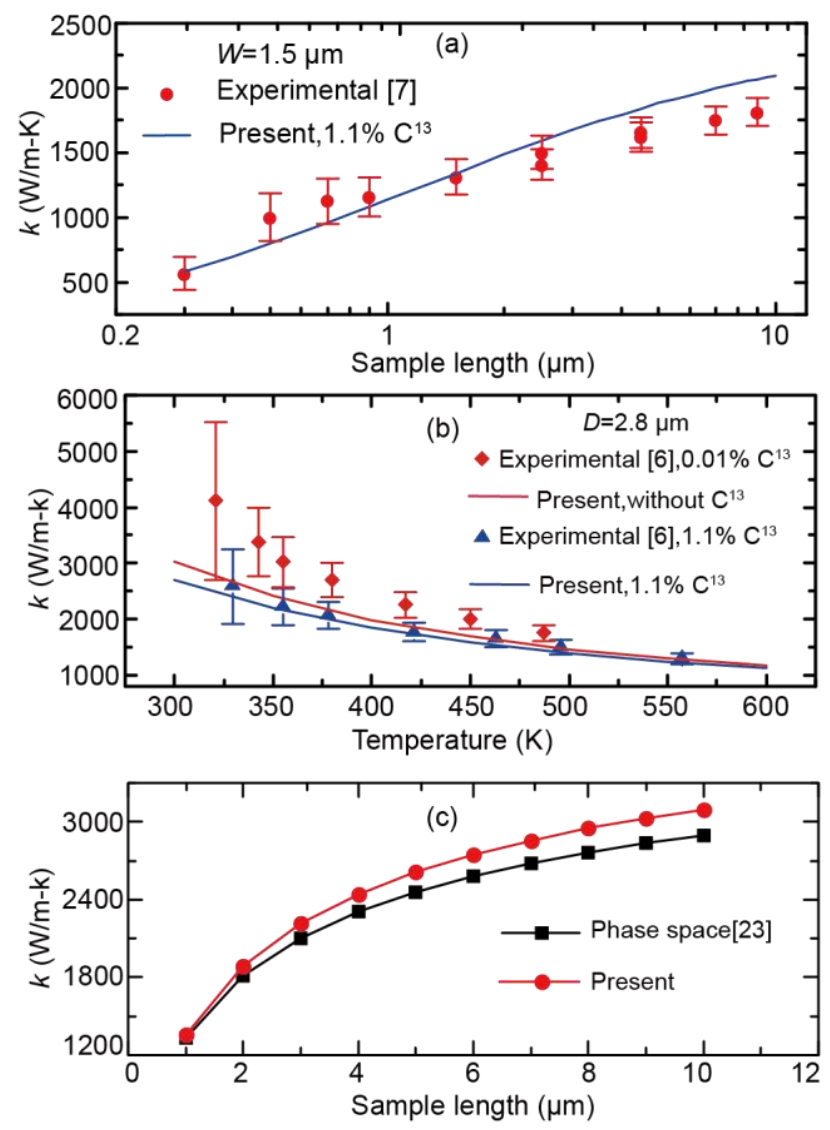

Fig. 3. (a) Comparison of calculated $k$ with previous experimental data for samples of a width $W=1.5 \mu \mathrm{m}$ and different lengths. (b) Comparison of calculated $k$ with previous experimental data for samples of a fixed diameter $D=2.8 \mu \mathrm{m}$ at different temperatures. (c) Comparison of calculated $k$ with previous results from a phase space approach $[23]$.

temperature $k$ for different sample lengths $L$ agree with recently measured data for suspended samples with a width $W=1.5 \mu \mathrm{m}$ [7]. Figure $3 \mathrm{~b}$ compares the calculated $k$ with experimental data from Ruoff's group [6] for graphene suspended over circular wells with a diameter $D=2.8 \mu \mathrm{m}$ at different temperatures. For samples with naturally occurring carbon isotopes, acceptable agreement is observed throughout the 
considered temperature range. For isotopically purified samples, a $\sim 12 \%$ enhancement in $k$ is predicted at room temperature, significantly less than the measured enhancement. Nonetheless, the calculated enhancement in $k$ falls within the experimental uncertainties [6] from the Raman technique. Recently, slight $k$ enhancements with isotopic purification, $\sim 13 \%$ and $\sim 16 \%$, were also reported by Lindsay et al. [23] and Fugallo et al. [28], respectively. The reason for this discrepancy is unknown yet, and requires further experimental validation from more accurate measurements. We note that indeed there are discrepancies among $k$ measurements in the literature. Here we compared to two independent sets of data (separate microbridge and Raman thermometry measurements) to give some confidence in the calculations. Our calculations may not agree with other measured data for which other extrinsic factors may be more important. The phase space approach [23] is also used to calculate room temperature $k$ of finite samples based on the same IFCs and considering boundary scattering with the form $\frac{1}{\tau_{\lambda}^{b}}=\frac{2\left|v_{\lambda}^{x}\right|}{L}$ and phonon-isotope scattering from natural isotope mass disorder. The corresponding results are shown in Fig.3c and compared with those by the present methodology. The agreement is quite good, somewhat surprising given the significant differences in algorithms implemented in the two methods.

\section{3 . Role of ZA modes for the thermal transport at different temperatures}

To better understand the role of intrinsic three-phonon scattering, we calculate the frequency $\omega_{\lambda}$ dependent normalized $k$ accumulation for unstrained infinite graphene at 
$300 \mathrm{~K}$. The values are normalized by the $k$ of unstrained, isotopically pure, infinite graphene, $5450 \mathrm{~W} / \mathrm{m}-\mathrm{K}$. As shown in Figure 4a, ZA phonons are the main heat carriers in suspended unstrained graphene, contributing about $88 \%$ to $k$ at room temperature. Moreover, the relative contribution of ZA phonons to $k$, as denoted by $k_{\mathrm{ZA}} / k$ at given $T$ is shown in Figure $4 \mathrm{~b}$. Although increasing $T$ decreases $k_{\mathrm{ZA}} / k$, the dominant contribution of ZA phonons is still obvious (above 80\%) for temperatures from $200 \mathrm{~K}$ to $2000 \mathrm{~K}$. We also see that the intrinsic $k$ shows different temperature dependent behaviors: $k \propto T^{-1.13}+6 \times 10^{6} T^{-3.96}$ for $T \leq 1200 \mathrm{~K}$ and $k \propto T^{-1.05}$ for $T>1200 \mathrm{~K}$. On the other hand, the absorption process $\mathrm{ZA}+\mathrm{ZA} \rightarrow \mathrm{TA}$ (LA) [22] dominates the scattering of ZA modes and results in decreasing and increasing $k$ contributions of ZA and TA(LA) modes, respectively, with increasing $N_{1}$ until convergence. For strained graphene, ZA phonons also provide the dominant contributions to $k$ over the considered temperature range and $k$ still diverges at temperature up to $2000 \mathrm{~K}$, as seen in Figure $4 \mathrm{c}$, demonstrating that 

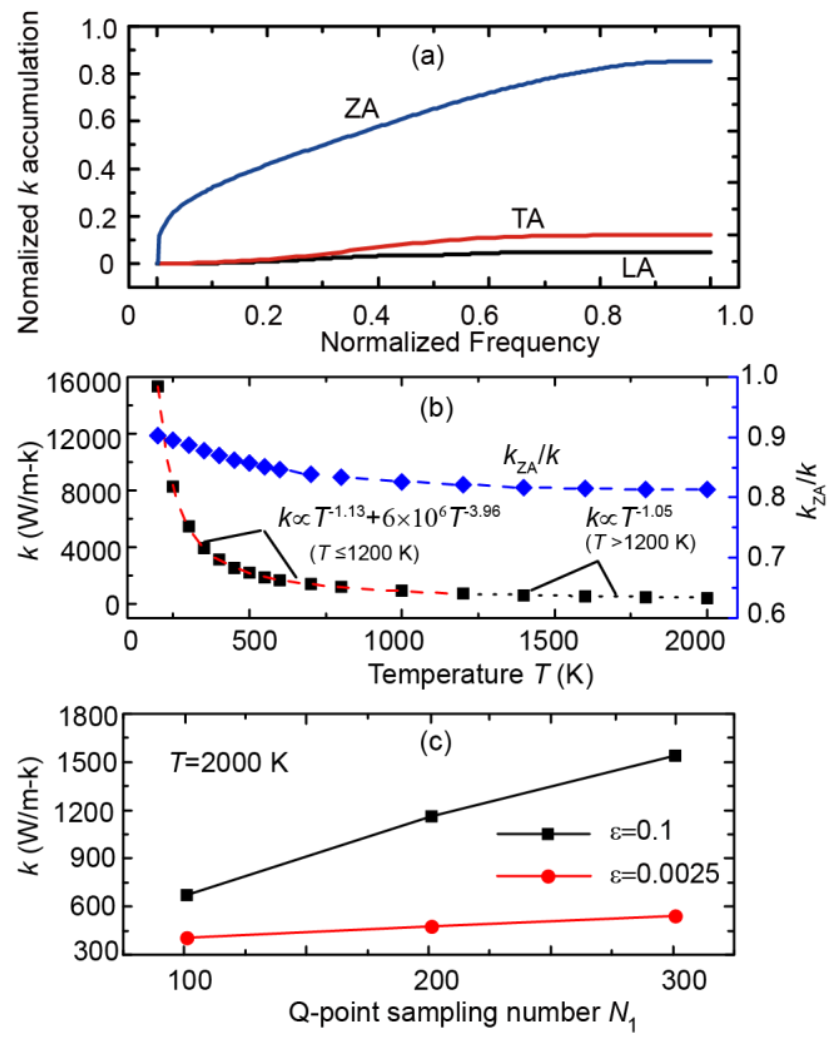

Fig. 4. (a) Normalized $k$ accumulations of acoustic phonons for unstrained graphene with respect to frequency normalized by the corresponding cutoff frequency, i.e., 16.1 THz, 23.5 THz and 40.4 THz for ZA, TA and LA branches, respectively. (b) Effects of temperature on the intrinsic $k$ and the contribution of ZA phonons. (c) Convergence of $k$ with $q$-point sampling density for isotropic tensile strains $\varepsilon=0.0025$ and $\varepsilon=0.1$ at temperature $T=2000 \mathrm{~K}$.

that even at high temperature (though below the Debye temperature $2100 \mathrm{~K}$ ) the intrinsic $k$ does not converge within the three-phonon scattering framework though these scattering rates increase significantly. As explained by Lindsay et al. [19, 23], this dominance of the ZA phonons arises because the reflection symmetry of graphene forbids three-phonon scatterings involving odd numbers of ZA phonons. This 
manifests itself in the scattering calculations via matrix elements that are zero for these couplings. This gives another restriction to the scattering phase space beyond crystal momentum and energy conservation. Previous experiments on supported graphene $[12,13]$ also attribute measured reductions in $k$ to the suppression of ZA phonon contributions by substrate coupling. Therefore, engineering $k$ by suppressing the ZA phonon contributions through substrate coupling $[12,13]$, irregular doping or defect distributions [11, 28], or irregular out-of-plane deformations may be worthwhile. The most recent work [46] only investigated the temperature effects on phonon thermal transport in unstrained graphene under $800 \mathrm{~K}$, which is still within the low-temperature range considering the graphene Debye temperature is up to $2100 \mathrm{~K}$. Therefore, the present investigations may further the understanding of temperature effects. We note that at high temperature the $k$ behavior of strained graphene may be affected by higher order phonon-phonon scattering [22]. A recent work [47] has demonstrated the importance of four-phonon scattering in bulk materials at high temperature.

\section{4 . Interplay of size and strain effects}

The ratios of phonon transport lifetimes to relaxation times given by SMRTA solutions show significant amplification for most acoustic modes, demonstrating that the SMRTA severely overestimates the intrinsic resistance in graphene. Therefore, using the SMRTA to determine size-dependent $k$ may be misleading as the boundary scattering is relatively weak in comparison [48]. To examine further the mechanism for the significant size effects observed in previous experiments [7] and numerical 
calculations $[23,28,29]$, we plot the normalized $k$ accumulation with respect to the mean free path (MFP) (Figure 5a) and phonon wavelength (Figure 5b) of acoustic phonons in infinite unstrained graphene without isotope scattering. The contributions from ZA phonons with ultra-long MFP saturate at about $8 \mathrm{~cm}$, while those of in-plane TA and LA phonons saturate around $10 \mu \mathrm{m}$ or less. Therefore, for suspended samples with the system sizes of several hundreds of microns or less, boundary scattering will
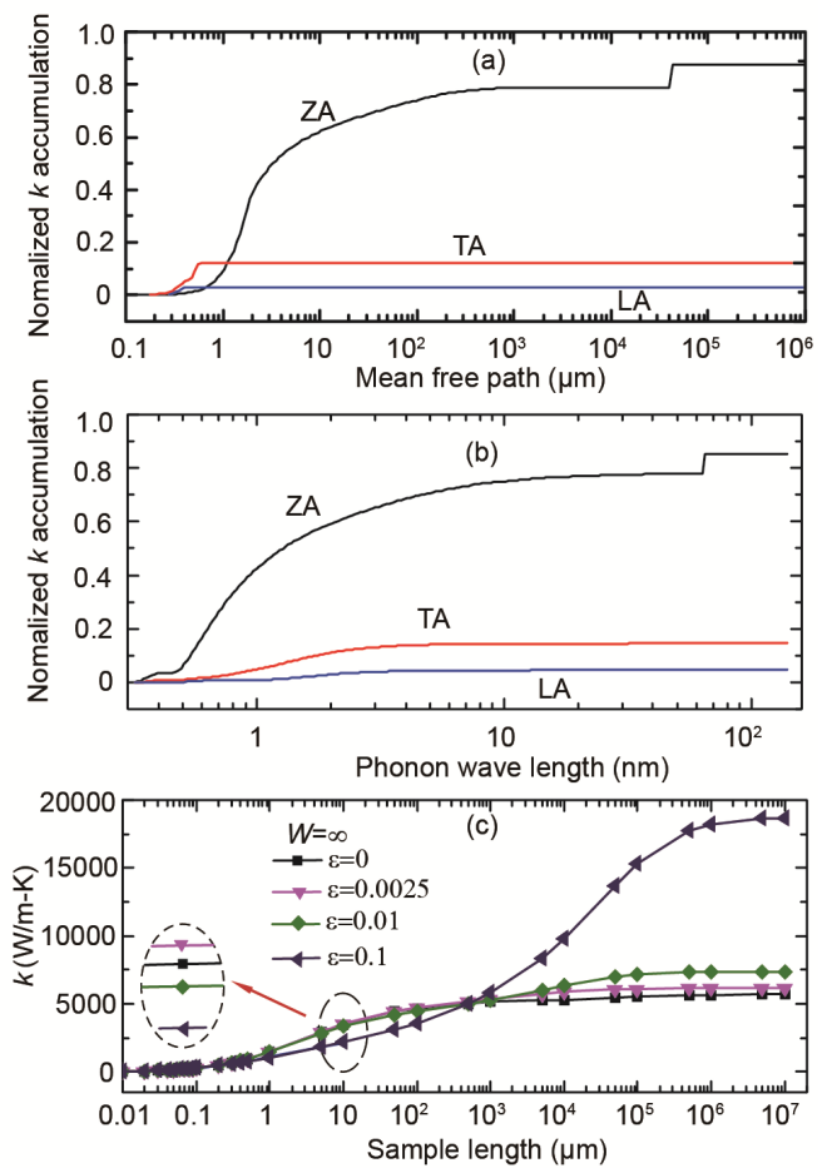

Fig. 5. (a) Normalized $k$ accumulation with respect to the phonon mean free path for unstrained graphene. (b) Normalized $k$ accumulation with respect to the phonon wavelength for unstrained graphene. (c) Effects of sample length and strain on $k$. 
significantly limit the thermal conductivity, and the calculated $k$ will increase remarkably with increasing system size as demonstrated by the length dependent $k$ shown in Figure $5 \mathrm{c}$ corresponding to the case $\varepsilon=0, W=\infty$. Moreover, our calculation results as shown in Figure $5 \mathrm{~b}$ demonstrate that phonons with a wavelength smaller than $10 \mathrm{~nm}$ contribute $\sim 80 \%$ to the intrinsic $k$, indicating low-frequency long-wave phonon contributions are not as important as expected before [6, 7]. However, for strained graphene, as shown in Fig.1a, the divergence of $k$ with decreasing $q$ or increasing size means the low-frequency long wavelength ZA modes play a dominant role in determining thermal transport. Therefore, the tensile strain acts as a key to turn on the long wavelength effect. We note that previous MD simulations $[18,49]$ also showed the divergence under strain and the dominant contributions from ZA modes. Recently, we systematically investigated strain effects on thermal transport of buckled group-IV monolayers including silicene, germanene and stanene [50] under tensile strains. A strong size effect on $k$ for unstrained buckled group-IV monolayers was found. Similar conclusions were found by Xie et al. [51] for silicene that tensile strains may significantly enhance $k$ with a peak enhancement at strain $\sim 0.04$. Contrasting with the ignorable size effect of $k$ for unstrained silicene, the size effect for unstrained graphene is up to $\sim 8 \mathrm{~cm}$, indicating the significant effect from the buckled backbone of silicene.

To illuminate tensile strain effects on $k$ of finite graphene samples, i.e., the interplay between strain and size effects, we also plot the sample length $L$ dependent room-temperature $k$ at different strain levels in Figure 5c. Within the applicable length range $1 \mu \mathrm{m}<L \leq 500 \mu \mathrm{m}$, a rather small strain $(\varepsilon=0.0025)$ leads to a slightly higher $k$ 
compared with that of the unstrained case, while further increasing strain results in decreasing $k$, as shown in the inset. Lindsay et al. [23] also observed this behavior for a $10-\mu \mathrm{m}$ system. However, within this length range, the difference in $k$ caused by a strain less than 0.01 is estimated to be less than $10 \%$. Considering the uncertainties involved in experimental measurements $(\sim 20 \%)$, it is difficult to distinguish the strain effects as strains during measurements are often smaller than 0.01 . For $L>500 \mu \mathrm{m}$, the trend changes, i.e., larger strain gives higher $k$ and several times improvement in $k$ by strain for large samples can be achieved. Considering the large graphene fracture strain ( 0.25) and recently successfully fabrication [34] of single crystal graphene sample with the size of a few centimeters, the strong dependence of $k$ on strain and size for large graphene samples indicates the great potential in tuning $k$ of graphene by varying size and strain. The strong dependence of $k$ on strain magnitude and sample size results from a competition between boundary and intrinsic three-phonon scattering. Our analysis shows that the mode heat capacities of ZA phonons decrease due to the decrease of phonon number per unit frequency. For a ZA mode $\lambda$, its contribution $k_{\lambda}^{\text {total }}$ to $k$ satisfies $1 / k_{\lambda}^{\text {total }}=1 / k_{\lambda}^{\text {intrinsic }}+1 / k_{\lambda}^{\text {boundary }}$, where $1 / k_{\lambda}^{\text {intrinsic }}$ and $1 / k_{\lambda}^{\text {boundary }}$ are the thermal resistivities corresponding to the intrinsic phonon-phonon scattering and boundary scattering, respectively. At a fixed sample length, increasing strain decreases $k_{\lambda}^{\text {boundary }}$ due to the decrease in mode heat capacities of ZA phonons while enhancing $k_{\lambda}^{\text {intrinsic }}$ due to the increase in lifetimes of ZA phonons. Therefore, for applicable sample sizes in the range $1 \mu \mathrm{m}<L \leq 500 \mu \mathrm{m}$ for which $k_{\lambda}^{\text {intrinsic }}$ and $k_{\lambda}^{\text {boundary }}$ are comparable, their opposite variation trends will result in a peak 
enhancement to $k_{\lambda}^{\text {total }}$ by strain, as observed in $k$. For $L>500 \mu \mathrm{m}$, we find intrinsic three-phonon scattering dominate over boundary scattering for the whole frequency range considered. Therefore, increasing strain leads to the improvement of $k_{\lambda}^{\text {total }}$ and in turn $k$. This result is consistent with those shown in Figure 1a for which the boundary scattering is neglected completely. Interestingly, the tensile-strain-induced enhancement of $k$ is in contrast to those reported for other carbon-based materials such as 3D diamond [52] and 1D carbon nanotubes [30,53], wherein tensile strains reduce $k$ through phonon softening [53]. While the softening of LA, TA and optic phonons in graphene is indeed observed here (see Fig.2), we find hardening of the ZA modes, i.e., higher ZA frequencies and group velocities of low frequency modes for the strain levels considered. The ZA phonon hardening coupled with decreasing anharmonic IFCs increases the lifetimes of ZA phonons and enhances $k$. We note that, in calculations for strained graphene going from finite size to infinity, using the intrinsic three-phonon scattering rates from a finite $q$-sampling density will result in a false convergence for $k$, as shown by cases $\varepsilon=0.0025$ and 0.01 in Figure 5c. Theoretically, $N_{1} \rightarrow \infty$ is required to match $L \rightarrow \infty$ and present the divergence for strained graphene. Our tests using a larger $q$-sampling density $\left(N_{1}>301\right)$ give the uniform results in Figure 5c except that the false convergence plateau for $k$ occurs at a higher $k$ value and larger length, validating our judgments on $k$ variations with size and strain. In other words, the actual tuning range for $k$ of finite graphene is expected to be even larger than the results shown in Figure 5c. 


\section{Conclusive remarks}

Based on rigorous first-principles lattice dynamics calculations we present a comprehensive picture of phonon thermal transport in unstrained and strained graphene. Acceptable agreement between calculated $k$ and experimental data as well as agreement with results from a previous phase space methodology validate the present approach for calculating $k$ in $2 \mathrm{D}$ systems. We find that the intrinsic room temperature $k$ converges for unstrained graphene but diverges for strained graphene with system size. Analysis based on the phonon lifetimes confirms this finding. For unstrained graphene, we conclude that centimeter-order MFP of ZA phonons is responsible for the significant size effect observed in previous experiments. Lowfrequency long wavelength ZA phonons are not as important as expected before in unstrained graphene while they play the dominant role in strained graphene for thermal transport below $2000 \mathrm{~K}$. For finite strained graphene, tensile strain hardens the flexural modes, increases their lifetimes and causes unusual dependences of thermal conductivity on sample size and strain due to the competition between the boundary and phonon-phonon scattering; once the sample larger than $500 \mu \mathrm{m}$, increasing strain or size may effectively enhance $k$.

\section{Acknowledgements}

We are thankful for the financial support from the Hong Kong General Research Fund under Grant Nos. 623212, 613413 and 152140/14E, the Hong Kong Polytechnic University under Grant No. 1-99QP and the National Natural Science Foundation of 
China under Grant No. 51271157. L. L. acknowledges support from the U. S. Department of Energy, Office of Science, Office of Basic Energy Sciences, Materials Sciences and Engineering Division and the National Energy Research Scientific Computing Center (NERSC), a DOE Office of Science User Facility supported by the Office of Science of the U. S. Department of Energy under Contract No. DE-AC02$05 \mathrm{CH} 11231$.

\section{References}

[1] A.A. Balandin, S. Ghosh, W. Bao, I. Calizo, D. Teweldebrhan, F. Miao, C.N. Lau, Superior thermal conductivity of single-layer graphene, Nano Lett, 8(3) (2008) 902907.

[2] Y. Wang, A. Vallabhaneni, J. Hu, B. Qiu, Y.P. Chen, X. Ruan, Phonon Lateral Confinement Enables Thermal Rectification in Asymmetric Single-Material Nanostructures, Nano Lett, 14(2) (2014) 592-596.

[3] E. Pop, V. Varshney, A.K. Roy, Thermal properties of graphene: Fundamentals and applications, Mrs Bull, 37(12) (2012) 1273-1281.

[4] A.A. Balandin, Thermal properties of graphene and nanostructured carbon materials, Nat Mater, 10(8) (2011) 569-581.

[5] S. Chen, A.L. Moore, W. Cai, J.W. Suk, J. An, C. Mishra, C. Amos, C.W. Magnuson, J. Kang, L. Shi, Raman measurements of thermal transport in suspended monolayer graphene of variable sizes in vacuum and gaseous environments, Acs Nano, 5(1) (2010) 321-328.

[6] S. Chen, Q. Wu, C. Mishra, J. Kang, H. Zhang, K. Cho, W. Cai, A.A. Balandin, R.S. Ruoff, Thermal conductivity of isotopically modified graphene, Nat Mater, 11(3) (2012) 203-207.

[7] X. Xu, L.F. Pereira, Y. Wang, J. Wu, K. Zhang, X. Zhao, S. Bae, C.T. Bui, R. Xie, J.T. Thong, Length-dependent thermal conductivity in suspended single-layer graphene, Nat Commun, 5 (2014) 3689.

[8] W. Cai, A.L. Moore, Y. Zhu, X. Li, S. Chen, L. Shi, R.S. Ruoff, Thermal transport in suspended and supported monolayer graphene grown by chemical vapor deposition, Nano Lett, 10(5) (2010) 1645-1651.

[9] J.-U. Lee, D. Yoon, H. Kim, S.W. Lee, H. Cheong, Thermal conductivity of suspended pristine graphene measured by Raman spectroscopy, Phys Rev B, 83(8) (2011) 081419. 
[10] C. Faugeras, B. Faugeras, M. Orlita, M. Potemski, R.R. Nair, A. Geim, Thermal conductivity of graphene in corbino membrane geometry, Acs Nano, 4(4) (2010) 1889-1892.

[11] M.T. Pettes, I. Jo, Z. Yao, L. Shi, Influence of polymeric residue on the thermal conductivity of suspended bilayer graphene, Nano Lett, 11(3) (2011) 1195-1200.

[12] Z. Wang, R. Xie, C.T. Bui, D. Liu, X. Ni, B. Li, J.T. Thong, Thermal transport in suspended and supported few-layer graphene, Nano Lett, 11(1) (2010) 113-118.

[13] J.H. Seol, I. Jo, A.L. Moore, L. Lindsay, Z.H. Aitken, M.T. Pettes, X. Li, Z. Yao, R. Huang, D. Broido, Two-dimensional phonon transport in supported graphene, Science, 328(5975) (2010) 213-216.

[14] Y.K. Koh, M.-H. Bae, D.G. Cahill, E. Pop, Heat conduction across monolayer and few-layer graphenes, Nano Lett, 10(11) (2010) 4363-4368.

[15] W. Jang, Z. Chen, W. Bao, C.N. Lau, C. Dames, Thickness-dependent thermal conductivity of encased graphene and ultrathin graphite, Nano Lett, 10(10) (2010) 3909-3913.

[16] A.D. Liao, J.Z. Wu, X. Wang, K. Tahy, D. Jena, H. Dai, E. Pop, Thermally limited current carrying ability of graphene nanoribbons, Phys Rev Lett, 106(25) (2011) 256801.

[17] W.-R. Zhong, M.-P. Zhang, B.-Q. Ai, D.-Q. Zheng, Chirality and thicknessdependent thermal conductivity of few-layer graphene: A molecular dynamics study, Appl Phys Lett, 98(11) (2011) 113107.

[18] L.F.C. Pereira, D. Donadio, Divergence of the thermal conductivity in uniaxially strained graphene, Phys Rev B, 87(12) (2013) 125424.

[19] L. Lindsay, D. Broido, N. Mingo, Flexural phonons and thermal transport in multilayer graphene and graphite, Phys Rev B, 83(23) (2011) 235428.

[20] D. Nika, E. Pokatilov, A. Askerov, A. Balandin, Phonon thermal conduction in graphene: Role of Umklapp and edge roughness scattering, Phys Rev B, 79(15) (2009) 155413.

[21] D. Singh, J.Y. Murthy, T.S. Fisher, Mechanism of thermal conductivity reduction in few-layer graphene, J Appl Phys, 110(4) (2011) 044317.

[22] N. Bonini, J. Garg, N. Marzari, Acoustic phonon lifetimes and thermal transport in free-standing and strained graphene, Nano Lett, 12(6) (2012) 2673-2678.

[23] L. Lindsay, W. Li, J. Carrete, N. Mingo, D. Broido, T. Reinecke, Phonon thermal transport in strained and unstrained graphene from first principles, Phys Rev B, 89(15) (2014) 155426.

[24] L. Paulatto, F. Mauri, M. Lazzeri, Anharmonic properties from a generalized third-order ab initio approach: Theory and applications to graphite and graphene, Phys Rev B, 87(21) (2013) 214303.

[25] B.D. Kong, S. Paul, M.B. Nardelli, K.W. Kim, First-principles analysis of lattice thermal conductivity in monolayer and bilayer graphene, Phys Rev B, 80(3) (2009) 033406.

[26] W.J. Evans, L. Hu, P. Keblinski, Thermal conductivity of graphene ribbons from equilibrium molecular dynamics: Effect of ribbon width, edge roughness, and hydrogen termination, Appl Phys Lett, 96(20) (2010) 203112.

[27] P. Klemens, Theory of thermal conduction in thin ceramic films, Int $\mathbf{J}$ Thermophys, 22(1) (2001) 265-275. 
[28] G. Fugallo, A. Cepellotti, L. Paulatto, M. Lazzeri, N. Marzari, F. Mauri, Thermal conductivity of graphene and graphite: collective excitations and mean free paths, Nano Lett, 14(11) (2014) 6109-6114.

[29] M. Park, S.-C. Lee, Y.-S. Kim, Length-dependent lattice thermal conductivity of graphene and its macroscopic limit, J Appl Phys, 114(5) (2013) 053506.

[30] X. Li, K. Maute, M.L. Dunn, R. Yang, Strain effects on the thermal conductivity of nanostructures, Phys Rev B, 81(24) (2010) 245318.

[31] D.L. Nika, A.A. Balandin, Two-dimensional phonon transport in graphene, J Phys: Condens Mat, 24(23) (2012) 233203.

[32] N. Mounet, N. Marzari, First-principles determination of the structural, vibrational and thermodynamic properties of diamond, graphite, and derivatives, Phys Rev B, 71(20) (2005) 205214.

[33] A. Ward, D. Broido, D.A. Stewart, G. Deinzer, Ab initio theory of the lattice thermal conductivity in diamond, Phys Rev B, 80(12) (2009) 125203.

[34] Y. Hao, M. Bharathi, L. Wang, Y. Liu, H. Chen, S. Nie, X. Wang, H. Chou, C. Tan, B. Fallahazad, The role of surface oxygen in the growth of large single-crystal graphene on copper, Science, 342(6159) (2013) 720-723.

[35] W. Li, J. Carrete, N.A. Katcho, N. Mingo, ShengBTE: A solver of the Boltzmann transport equation for phonons, Comput Phys Commun, 185(6) (2014) 1747-1758.

[36] P. Giannozzi, S. Baroni, N. Bonini, M. Calandra, R. Car, C. Cavazzoni, D. Ceresoli, G.L. Chiarotti, M. Cococcioni, I. Dabo, QUANTUM ESPRESSO: a modular and open-source software project for quantum simulations of materials, $\mathrm{J}$ Phys.: Condens Matt, 21(39) (2009) 395502.

[37] L. Lindsay, D. Broido, T. Reinecke, Ab initio thermal transport in compound semiconductors, Phys Rev B, 87(16) (2013) 165201.

[38] M. Mohr, J. Maultzsch, E. Dobardžić, S. Reich, I. Milošević, M. Damnjanović, A. Bosak, M. Krisch, C. Thomsen, Phonon dispersion of graphite by inelastic X-ray scattering, Phys Rev B, 76(3) (2007) 035439.

[39] J. Maultzsch, S. Reich, C. Thomsen, H. Requardt, P. Ordejón, Phonon dispersion in graphite, Phys Rev Lett, 92(7) (2004) 075501.

[40] N. Mingo, D. Broido, Length dependence of carbon nanotube thermal conductivity and the "problem of long waves", Nano Lett, 5(7) (2005) 1221-1225.

[41] L. Lindsay, D. Broido, N. Mingo, Lattice thermal conductivity of single-walled carbon nanotubes: Beyond the relaxation time approximation and phonon-phonon scattering selection rules, Phys Rev B, 80(12) (2009) 125407.

[42] L.N. Virgin, Vibration of axially-loaded structures, Cambridge University Press, 2007.

[43] J. Carrete, W. Li, L. Lindsay, D.A. Broido, L.J. Gallego, N. Mingo, Physically founded phonon dispersions of few-layer materials and the case of borophene, Mater. Res Lett, (2016) 1-8.

[44] J. Turney, A. McGaughey, C. Amon, In-plane phonon transport in thin films, J Appl Phys, 107(2) (2010) 024317.

[45] K. Esfarjani, G. Chen, H.T. Stokes, Heat transport in silicon from first-principles calculations, Phys Rev B, 84(8) (2011) 085204.

[46] A. Cepellotti, G. Fugallo, L. Paulatto, M. Lazzeri, F. Mauri, N. Marzari, Phonon hydrodynamics in two-dimensional materials, Nat Commun, 6 (2015) 6400. 
[47] T. Feng, X. Ruan, Quantum mechanical prediction of four-phonon scattering rates and reduced thermal conductivity of solids, Phys Rev B, 93(4) (2016) 045202.

[48] L. Lindsay, D. Broido, N. Mingo, Diameter dependence of carbon nanotube thermal conductivity and extension to the graphene limit, Phys Rev B, 82(16) (2010) 161402.

[49] M. Gill-Comeau, L.J. Lewis, Heat conductivity in graphene and related materials: A time-domain modal analysis, Phys Rev B, 92(19) (2015) 195404.

[50] Y. Kuang, L. Lindsay, S. Shi, G.-P. Zheng, Tensile strains give rise to strong size effects for thermal conductivities of silicene, germanene and stanene, Nanoscale, 8(2016) 3760-3767.

[51] H. Xie, T. Ouyang, É. Germaneau, G. Qin, M. Hu, H. Bao, Large tunability of lattice thermal conductivity of monolayer silicene via mechanical strain, Phys Rev B, 93(7) (2016) 075404.

[52] D. Broido, L. Lindsay, A. Ward, Thermal conductivity of diamond under extreme pressure: a first-principles study, Phys Rev B, 86(11) (2012) 115203.

[53] Z. Xu, M.J. Buehler, Strain controlled thermomutability of single-walled carbon nanotubes, Nanotechnology, 20(18) (2009) 185701. 